\title{
Review
}

\section{Electronic Cigarettes: A Short Review}

\author{
J.F. Bertholon a, b, e M.H. Becquemin ${ }^{\text {a, c }}$ I. Annesi-Maesanod, e B. Dautzenberg ${ }^{b}$ \\ a Laboratoire de Granulométrie et de Dépôt des Aérosols, Assistance Publique Hôpitaux de Paris, \\ bUniversité Pierre et Marie Curie, Paris 6, ' Université Denis Diderot et UPRES 2397, ${ }^{\mathrm{d}}$ Institut National de la Santé et \\ de la Recherche Médicale, Unité 707, and ' Department of Epidemiology of Allergic and Respiratory Diseases, \\ Unité de Recherche Mixte en Santé, Paris, France
}

\section{Key Words}

Electronic cigarettes · Propylene glycol · Nicotine

\begin{abstract}
Marketed since 2004 as an alternative to nicotine delivery and advertised as a valid means to smoking cessation, the electronic (e)-cigarette has been the subject of much controversy but very little experimental study. This review provides a brief summary of the current knowledge of this product. Propylene glycol and glycerol, the main ingredients of the fluid that is vaporized, have proved to be harmless in the fog machines of the entertainment industry. However, in the case of the e-cigarette fluid, the composition is not properly labeled: additives like nicotine and flavors vary between and within brands and contamination with various chemicals has been detected. The short-term toxicity seems low, but the long-term toxicity is unknown. The usefulness of the e-cigarette in smoking cessation has still to be clinically established.
\end{abstract}

(C) 2013 S. Karger AG, Basel

\section{Introduction}

First manufactured in China (2003) and widely available since, the electronic (e)-cigarette consists of a battery, a microchip, a red LED simulating a burning cigarette tip, a cartridge containing a liquid (propylene glycol in water) and a vaporization chamber, which produce an aerosol that simulates the smoke of a cigarette (fig. 1).

Nicotine and other additives like flavorings and glycerol (purified vegetable glycerine) may be added in various concentrations to the liquid. No combustion is involved in the process and the 'smoke' produced is an aerosol of liquid particles, a 'mist' [1].

Advertised as a valid way to quit smoking real cigarettes, the e-cigarette has been the subject of much controversy but little experimental study. This review provides a brief overview of the current evidence on the ecigarette.

\section{Aerosol Generation}

Besides water, the main ingredient of the e-liquid is usually propylene glycol (PG) in water. A mechanical sensor detects when the user inhales and it then triggers the microchip controlling the heater which raises the temperature in the vaporization chamber.

As specified by the manufacturers, the temperatures in the vaporization chamber range from 40 to $65^{\circ} \mathrm{C}$ [1]. Recently, however, variable voltage devices gained popularity as they can increase the temperature of operation and the heater surface area in order to tune the vapor to the user's satisfaction. No reliable data could be found on the range of temperatures in these recent devices. The same uncertainty exists about the operating temperature in

\section{KARGER}

E-Mail karger@karger.com www.karger.com/res
2013 S. Karger AG, Basel

0025-7931/13/0865-0433\$38.00/0
Jean-François Bertholon

Département de Physiologie

UPMC - 27, rue de Chaligny

FR-75012 Paris (France)

E-Mail jean-francois.bertholon@upmc.fr 
Fig. 1. Components of an e-cigarette (redrawn from [1]).

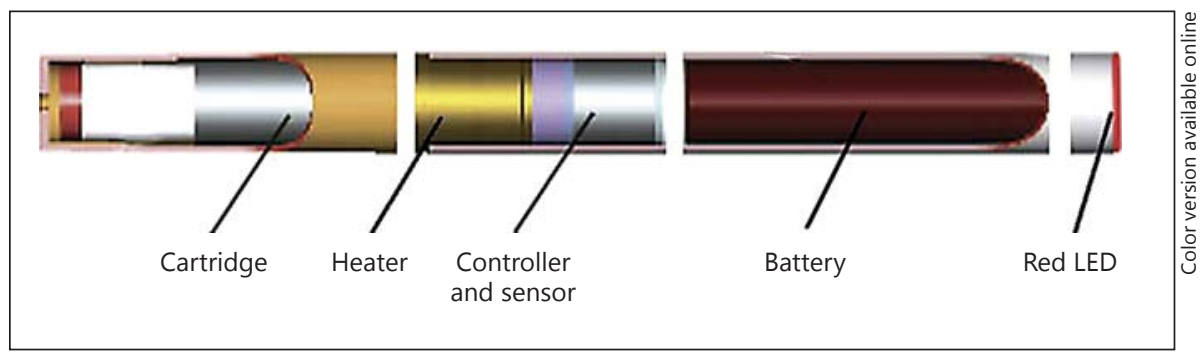

chambers vaporizing PG-glycerol or glycerol-only fluids as glycerol vaporizes at a higher temperature. Some manufacturers claim they limit the temperature to $<100^{\circ} \mathrm{C}$ to avoid the degradation into toxic acrolein which occurs at higher temperatures.

The aerosol is generated by vaporization at these temperatures and the vapor then condenses into a visible fog. The principle is the same as in the mist generators of theaters, discotheques or movie sets. The droplets of PG or glycerol in the fog carry the chemical substances, added for taste and flavor, and an optional dose of nicotine. The red LED at the tip of the cigarette is activated during inhalation. To produce the aerosol, a higher vacuum (suction) is required for an e-cigarette than for a conventional cigarette. With each successive puff, an even higher vacuum is required [2].

A review of manufacturer sites shows that a cartridge of e-liquid should generate between 10 and 250 puffs of aerosol which would be equivalent to 5-30 cigarettes. In fact, as the vapor is much less irritating to the airways than tobacco smoke, it need not be mixed with air and the puffs are greater in volume with e-cigarettes.

\section{Aerosol Characteristics}

The size of the aerosol particles produced by e-cigarettes has only recently been measured. Unlike a real cigarette, the e-cigarette does not spontaneously produce 'smoke' (secondary or side-stream), so only primary smoke (inhaled) and tertiary smoke (exhaled) have been studied: the mass median aerodynamic diameter (MMAD) of the primary current is about $600 \mathrm{~nm}$ and that of the tertiary current is about $300 \mathrm{~nm}$. Thus the particles are bigger than the tobacco-smoke particles from cigarettes $[3,4]$.

Pellegrino et al. [5] found that the concentration of fine and ultrafine particles in the aerosol produced by an e-cigarette was lower than with conventional cigarettes, but such a comparison is questionable as the time the ecigarette aerosol is in the air is very short.
Schripp et al. [6] have demonstrated the fast-changing nature of the aerosol particle size spectrum in a small test chamber $\left(8 \mathrm{~m}^{3}\right)$, while Bertholon et al. [3] showed that the half-life of PG e-cigarette 'smoke' in ambient air is around $10 \mathrm{~s}$, which is 100 times less than with cigarette smoke. This is caused by the rapid revaporization of the aerosol droplets at the ambient temperature. This was confirmed by Ingebrethsen et al. [7] who found different particles sizes when using different dilutions of the aerosol prior to measurement. Their results with a spectral transmission procedure found the particle diameters of the undiluted e-cigarette aerosol to be in the range of $250-450 \mathrm{~nm}$.

\section{Properties of PG}

PG (or propane-1,2-diol/1,2-dihydroxypropane/meth$\mathrm{yl}$ glycol) is an alcohol that is currently used as:

- an additive in food and cosmetics (E1520) as a humidifier and emulsifier. As such, it is also used to prevent tobacco drying out.

- an antifreeze that is noncorrosive and nontoxic as a replacement for ethylene glycol.

- a solvent in many pharmaceuticals (oral, injectable and topical) for substances that are insoluble in water (e.g. benzodiazepines and phenytoin). Due to its affinity to water, it has also been used in aerosolized drug-delivery systems like metered-dose inhalers and nebulizers.

Most of the available data on this product come from studies aimed at regulating the use of theatrical mist machines. In these too, glycerol may be added to PG in various proportions in order to lengthen the life of the aerosol in the air.

These mist generators are used to create special effects in theaters, discotheques and movies, to simulate fires for firefighter training or in cleaning and pest control enterprises.

According to studies conducted by the UK Atomic Energy Authority, the aerosol produced has an MMAD between 0.2 and $60 \mu \mathrm{m}$ depending on the generator. 


\section{Toxicity of PG}

PG is absorbed into the small intestine and is transformed by glycolysis into energetic compounds such as pyruvic and lactic acids, or by taking the path of ethanol, it yields acetic acid and propionic aldehyde (toxic). Nonmetabolized PG is excreted in urine either directly or after hepatic glucuronidation. Its half-life is $2 \mathrm{~h}$ in the blood and $4 \mathrm{~h}$ in the body. Acute lethal dosage (LD50) is approximately $20 \mathrm{~g} / \mathrm{kg}$ (small mammals) [8]. Long-term toxicity by ingestion and inhalation has not been observed in the rat and there is no evidence of carcinogenic or genotoxic action [8-13]. As PG is a di-alcohol, it could theoretically induce a state of intoxication.

PG is combustible and explosive air-vapor mixtures can form above $99^{\circ} \mathrm{C}$. These are not concerns for the user, but rather for the manufacturer due to the high concentrations $(2.6-12.6 \%)$ and temperatures required in the manufacturing process. Oxidation of PG at a high temperature yields the moderately toxic propionaldehyde and lactic, pyruvic and acetic acids.

According to the security data that must be filed by the manufacturer for marketing a chemical substance in Europe (REACH), PG is nontoxic upon inhalation and is slightly irritating to the skin. No limit in concentration for professionals (actors) has been set by France or the USA, while the UK has set the total limit for vapor plus particles to $474 \mathrm{mg} / \mathrm{m}^{3}$.

\section{Theatrical Mists}

At the request of actors' organizations, 2 studies were carried out by the Health Hazard Evaluation and by the Department of Community and Preventive Medicine of the Mount Sinai School of Medicine in the USA to examine the effects of PG-produced artificial smoke on professional actors in Broadway shows. Both studies concluded that there is irritation of the airways when the exposure is massive and prolonged, this effect being more significant in people with asthma. Another study, by the University of British Columbia in Canada, found mild but persistent modifications of the pulmonary function of staff that were chronically exposed to PG aerosols $[14,17]$. However, the FDA (USA) classified the artificial mists 'generally considered as safe'.

The Entertainment Services and Technology Association released a standard (ANSI E1.5) concerning the use of theatrical mists for healthy workers and audiences $[18$, 19]. This standard takes into consideration the concentration of particles in air but not the duration of exposure. In this respect, the comparison of the long-term toxicity of theatrical mists and that of e-cigarettes becomes partly irrelevant due to the very different patterns of exposure to the products.

\section{$P G$ and Pediatric Medicine}

Cases of acute intoxication by PG are rare and result from accidental ingestion of intravenous administration PG, generally in children. The required doses of PG $(>1 \mathrm{~g} /$ liter of plasma) cannot be attained using any existing delivery device. Nonetheless, the American Academy of Pediatrics has warned general practitioners and specialists about the use of PG in cosmetics and drugs for children. Various toxic effects have been observed: nervous system depression, hyperlactatemia, renal and respiratory problems, cardiac arrhythmia (1 case of cardiac arrest), hypotension, contact dermatitis (eczema), thrombophlebitis, overweight as well as growth anomalies in newborns [20-22]. However, according to the study and review of Lessman et al. [22], PG seems to have a low sensitization potential.

In France, the Direction générale de la santé (DGS) (http://www.senat.fr/rap/r07-176-1/r07-176-11.pdf) and the French National Institute for Industrial Environment and Risks (INERIS; http://www.inrs.fr/demeter/ DEM\%20050.pdf) recommend caution, especially for breastfeeding women, due to the absence of data on the passage of PG in breastmilk.

\section{Toxicity of Glycerol}

Glycerol is used in place of PG in a few brands of ecigarette or it is mixed with PG to modify the life of the aerosol. Its higher vaporization temperature could make it generate some toxic acrolein, but it is otherwise widely used in the food and chemical industries and is nontoxic. Absorbed glycerol is metabolized in the liver into carbon dioxide and water or to form glucose and glycogen.

\section{Toxicity of Fluid Additives}

The basic PG and/or glycerol mixture produces a white, odorless vapor, so natural or artificial substances are added to give it flavor. These substances have been studied extensively because they are used in the food industry and for indoor fragrances and deodorants: some are considered to be toxic and a number of them are close to known carcinogens [23].

\section{Flavorings}

Electronic cigarettes incorporate aromatic substances imitating tobacco (pure or mentholated), fruit, vanilla, 
caramel and coffee, among others. Their nature is generally not specified other than in imprecise terms like 'vegetable flavoring', but they most likely belong to the family of food-flavoring substances. While most of them are approved for human consumption, no data are available concerning the short- and long-term effects of their inhalation. As with theatrical mists, some artificial flavorings have been shown to be cytotoxic [24].

Generally speaking, the chemical composition of e-liquid is not given. When information is available on the package insert, it is not complete. As an example, the brand Cigartex states the composition of the e-liquid as $75.5 \%$ glycerol with $24.5 \%$ additives, $8 \%$ of which are 'artificial flavors'.

\section{Nicotine}

Various nicotine concentrations (range $6-36 \mathrm{mg} / \mathrm{ml}$ ) are present in e-liquid. The content specified on the eliquid container (vial or cartridge) may be given as total cartridge content or concentration and labeled as low, medium or high. Varying nicotine amounts can be found in e-cigarettes labeled as having the same concentration [25], which makes comparisons with regular cigarettes (nicotine content given in milligrams/cigarette) difficult.

\section{Contaminants}

In 16 studies compiled by Cahn and Siegel [26], with gas-chromatography mass spectrometry on 9 different brands of e-liquid or e-cigarette aerosols, no dangerous substance in any significant concentration was found.

Some samples contain the toxic diethyleneglycol [1]. Schripp et al. [6] found 1,2-propanediol, 1,2,3-propanetriol, diacetin flavorings, in the gas-phase of the e-cigarette that they tested.

Some nicotine and tobacco-specific nitrosamines could be found in 'no nicotine' fluids. Schripp et al. [6] and McAuley et al. [9] analyzed e-cigarette aerosols in search of known tobacco-smoke pollutants (volatile organic compounds, carbonyls, polyaromatic hydrocarbons and diethylene glycol) and concluded that the very low concentration of these compounds does not constitute a threat to human health.

Traces of tadalafil and rimonabant have been detected by liquid chromatography and mass spectrometry in some brands [27]. Contamination by various compounds of e-fluids due to poor quality control and lack of external custody is bound to be common due to the present legal status of these products.

\section{Effects on Health}

\section{Active (First-Hand) Smoking}

Gennimata et al. [28] and Vakali et al. [29] conducted 2 studies on 37 and 32 subjects, respectively. They found a series of effects after smoking a single e-cigarette such as cough, sore throat, eye irritation and an increase in airways resistance and heart rate. They concluded that ecigarettes may harm the respiratory system. Vardavas et al. [30] also recorded an immediate increase in airways resistance after smoking an e-cigarette.

In a journal of the European Society of Cardiology, another Greek team published a study comparing the hemodynamic measurements and echocardiograms of smokers and e-cigarette users before and after smoking a regular cigarette or an e-cigarette with nicotine for $7 \mathrm{~min}$. The cardiovascular effects were significant only after smoking a regular cigarette. They hypothesized that absorption of nicotine into the circulatory system is less with an e-cigarette [31].

\section{Passive Smoking}

As previously mentioned, the very short life of the ecigarette aerosol in air greatly reduces the risk of occasional second-hand passive smoking. For this reason and due to the lack of obvious toxicity, some users choose to use e-cigarettes in public places where tobacco-smoking is banned.

As with theatrical mist machines and depending on the relative proportions of PG, water and glycerol, the aerosol lasts for between a few seconds and several hours (3-4h) in suspension. Based on the compounds analyzed, a recent study of some pollutants generated by tobaccosmoking concluded that, in comparison, e-cigarette use indoors can be considered harmless to human health [9].

If production of the aerosol is massive or chronic, secondary sedimentation generates a viscous coating on local surfaces. This obviously creates a risk of pollution of the environment, which has been coined as third-hand passive smoking [32].

\section{Long-Term Toxicity}

Long-term exposure to PG has been studied extensively with mist machines. The study of long-term exposure to and the inhalation of the vaporized fluid from e-cigarettes is lacking; however, this is due to their having appeared only recently and their present legal status. 


\section{Role of the Electronic Cigarette in Smoking Cessation}

The WHO (2008) has observed that no study demonstrating the e-cigarette as a therapy for smokers has been undertaken. The FDA and the French Agency for Food, Environmental and Occupational Health Safety (ANSES) do not recommend its use or free distribution on the market. In February 2012, the European Respiratory Society stated that, according to current information, it did not classify e-cigarettes as a safe alternative to smoking, particularly in places where conventional smoking is banned [33]. In 2012, the IATA recommended prohibiting the use of e-cigarettes on all airplanes. In Europe, except in Austria and Turkey, the e-cigarette is still unauthorized as a nicotine delivery device.

It has been shown that e-cigarettes can deliver nicotine effectively and hereby reduce the craving for cigarettes [34]. In the e-cigarette, nicotine of course exerts its usual pharmacological effects. So, prior to accepting the e-cigarette as a nicotine delivery device to replace the smoking of tobacco, it would be desirable to obtain consistent information about the nicotine content of the e-fluids, i.e. total cartridge content and concentration.

The primary e-cigarette aerosol is made up of fine particles, but its characteristics change very rapidly and no inference can be made about its penetration deep into the lung. The penetration of its components, particularly nicotine, into the blood, has still to be assessed by means of plasma and urine measurements.

The e-cigarette can satisfy the chemical and behavioral aspects of cigarette addiction. In this respect, it merely replaces the conventional cigarette and spares the smoker the inhalation of hundreds of harmful chemicals that are found in tobacco smoke.

As shown above, the basic e-cigarette vapor of waterPG with or without glycerol appears to be safe in both the short and long term, so any risk would arise from the added flavorings and fragrances, which are already a public health issue, as well as from possible contamination with other chemical substances due to a lack of quality control.

A recent study on 300 smokers presented at the last meeting of the Society for Research on Nicotine and Tobacco $[35,36]$ concluded that the use of e-cigarette reduces tobacco consumption and could be a help in smoking cessation, particularly with nicotine-containing e-liquid. However, this observation applied to a very short period. In addition, perpetuation of the behavioral aspect of smoking could also be thought of as facilitating smoking relapse when compared to attempts at smoking cessation via means which do not involve the inhalation of smoke.

Long-term and randomized studies are necessary if the question of the use of e-cigarettes in medical protocols is to be answered. To date, in protocols on smoking cessation, we have not found any data on the results of the smoking of e-cigarettes combined with other chemical aids like varenicline.

As a recognized aid to smoking cessation, e-cigarettes provide competition on the profitable market of nicotinedelivering devices and psychoactive drugs designed to fight this addiction. Taking this into account, one must be cautious about the results of research on e-cigarettes and consider the possible conflicts of interest on either side.

\section{Conclusion}

Manufacturers and brands of the e-cigarette have multiplied since it was first released onto the market by Golden Dragon Holdings in 2004. The aim is to replace the conventional cigarette which was made commercially available at the beginning of the 19th century.

Many smokers see the e-cigarette as a good way to quit smoking and many others as a tool with which to maintain their nicotine intake in public places where smoking tobacco is banned.

Until more evidence is available on the subject, the smoking of e-cigarettes should not be considered as safe. However, from our review of the literature and bearing in mind the long experience with theatrical mists, the shortterm toxicity can be considered to be very low - except for some individuals with reactive airways - and the longterm toxicity depends on the additives and contaminants in PG and/or glycerol.

However, as the use of these devices with or without nicotine is rapidly increasing, we are still in need of data about its efficacy for smoking cessation. The e-cigarette is not used medically anywhere, at least not officially. If it were, manufacturers and their products would naturally have to be subjected to appropriate controls.

\section{Financial Disclosure and Conflicts of Interest}

No disclosures. 


\section{References}

1 Westenberger BJ: Evaluation of e-cigarettes. Rockville, FDA Center for Drug Evaluation and Research, Division of Pharmaceutical Analysis, 2009.

-2 Trtchounian A, Williams M, Talbot P: Conventional and electronic cigarettes (e-cigarettes) have different smoking characteristics. Nicotine Tob Res 2010;12:905-912.

-3 Bertholon JF, Becquemin MH, Roy M, Roy F, Ledur D, Annesi Maesano I, Dautzenberg B: Comparaison de l'aérosol de la cigarette électronique à celui des cigarettes ordinaires et de la chicha. Rev Mal Respir, in press.

4 Becquemin MH, Bertholon JF, Attoui M, Roy F, Roy M, Dautzenberg B: Tailles particulaires de la fumée produite par six différents types de cigarettes. Rev Mal Respir 2007;24:845852.

5 Pellegrino RM, Tinghino B, Mangiaracina G, Marani A, Vitali M, Protano C, Osborn JF, Cattaruzza MS: Electronic cigarettes: an evaluation of exposure to chemicals and fine particulate matter (PM). Ann Ig 2012;24:279288.

6 Schripp T, Markewitz D, Uhde E, Salthammer $\mathrm{T}$ : Does e-cigarette consumption cause passive vaping? Indoor Air 2013;23:25-31.

7 Ingebrethsen BJ, Cole SK, Alderman SL: Electronic cigarette aerosol particle size distribution measurements. Inhal Toxicol 2012;24: 976-984.

$>8$ Werley MS, McDonald P, Lilly P, Kirkpatrick D, Wallery J, Byron P, Venitz J: Non-clinical safety and pharmacokinetic evaluations of propylene glycol aerosol in Sprague-Dawley rats and Beagle dogs. Toxicology 2011;287: 76-90.

$>9$ McAuley TR, Hopke PK, Zhao J, Babaian S: Comparison of the effects of e-cigarette vapor and cigarette smoke on indoor air quality. Inhal Toxicol 2012;4:850-857.

-10 Montharu J, Le Guellec S, Kittel B, Rabemampianina Y, Guillemain J, Gauthier F, Diot P, de Monte M: Evaluation of lung tolerance of ethanol, propylene glycol, and sorbitan monooleate as solvents in medical aerosols. J Aerosol Med Pulm Drug Deliv 2010;23:41-46.

11 UNEP: OECD SIDS Initial Assessment Report 2001: Propylene glycol. www.chem. unep.ch/irptc/sids/OECDSIDS/sidspub.html

12 Janusz Szajewski MD: Warsaw Poison Control Centre 1991: Propylene glycol (PIM 443). IPCS INChem, 2009.

13 Fiches Internationales de Sécurité Chimique: Propylène glycol. http://www.cdc.gov/niosh/ ipcsnfrn/nfrn0321.html
14 Burr GA, Van Gilder TJ, Trout DB, Wilcox TG, Driscoll R: NIOSH Health Hazard Evaluation HETA 90-0355-2499. Actor's Equity Association, 1994.

15 HSE Consulting and Sampling, Inc.: Literature_Review_for_Glycerol_and_Glycols.pdf. for Entertainment Services \& Technology Association. http://casaa.org/uploads/Literature_Review_for_Glycerol_and_Glycols.pdf

16 Moline JM, Golden AL, Highland JH, Wilmarth KR, Kao AS: Health Effects Evaluation of Theatrical Smoke, Haze and Pyrotechnics. Prepared for Actor's Equity Pension and Health Trust Funds, June 6, 2000

17 Varughese S, Teschke K, Brauer M, Chow Y, van Netten C, Kennedy SM: Effects of theatrical smokes and mists on respiratory health in the entertainment industry. Am J Ind Med 2005;47:411-418.

18 ANSI E1.5 Entertainment Technology: Theatrical fog made with aqueous solutions of diand trihydric alcohols F\&S/1997-3017r7, 2003.

19 Raymond GE, Cohen JM: Recommended Exposure Guidelines for Glycol Fogging Agents. Project No. 6070-1001, 1997.

20 Cate JC IV, Hedrick R: Propylene glycol intoxication and lactic acidosis. N Engl J Med 1980;303:1237.

21 Arulanantham K, Genel M: Central nervous system toxicity associated with ingestion of propylene glycol. J Pediatr 1978;93:515-516.

22 Lessman H, Schnuch A, Geier J, Uter W: Skinsensitizing and irritant properties of propylene glycol. Contact Dermatitis 200;553:247259.

23 Cone JE, Shusterman D: Health effects of indoor odorants. Environ Health Perspect 1991; 95:53-59.

-24 Bahl V, Lin S, Xu N, Davis B, Wang YH, Talbot P: Comparison of electronic cigarette refill fluid cytotoxicity using embryonic and adult models. Reprod Toxicol 2012;4:529537.

25 Cheah NP, Chong NW, Tan J, Morsed FA, Yee SK: Electronic nicotine delivery systems: regulatory and safety challenges: Singapore perspective. Tob Control 2012, E-pub ahead of print.

26 Cahn Z, Siegel M: Electronic cigarettes as a harm reduction strategy for tobacco control: a step forward or a repeat of past mistakes? J Public Health Policy 2011;32:16-31.

27 Hadwiger ME, Trehy ML, Ye W, Moore T, Allgire J, Westenberger B: Identification of amino-tadalafil and rimonabant in electronic cigarette products using high pressure liquid chromatography with diode array and tandem mass spectrometric detection. J Chromatogr A 2010;48:7547-7555.
28 Gennimata SA, Palamidas A, Kaltsakas G, Tsikrika S, Vakali S, Gratziou C, Koulouris N: Acute effects of e-cigarette on pulmonary function in healthy subjects and smokers. ERS Congress, Vienna, 2012.

29 Vakali S, Tsikrika S, Gennimata SA, Kaltsakas G, Palamidas A, Koulouris N, Gratziou C: Acute impact of a single e-cigarette smoking on symptoms, vital signs and airways inflammatory response. ERS Congress, Vienna, 2012.

30 Vardavas CI, Anagnostopoulos N, Kougias M, Evangelopoulou V, Connolly GN, Behrakis PK: Short-term pulmonary effects of using an electronic cigarette: impact on respiratory flow resistance, impedance, and exhaled nitric oxide. Chest 2012;141:1400-1406.

31 Farsalinos K, Tsiapras D, Kyzopoulos S, Savvopoulos M, Avramidou E, Vassilopoulou D, Voudris V: Acute effects of using an electronic nicotine-delivery device (e-cigarette) on myocardial function: comparison with the effects of regular cigarettes. http://www.escardio.org/about/press/esc-congress-2012/ press-conferences/Documents/presentations/konstantinos-farsalinos.pdf

32 Kuschner WG, Reddy S, Mehrotra N, Paintal HS: Electronic cigarettes and third-hand tobacco smoke: two emerging health care challenges for the primary care provider. Int J Gen Med 2011;4:115-120.

33 ERS statement on e-cigarettes 2012. http:// www.ersnet.org/news/item/4494-europeanrespiratory-society-statement-on-e-cigarettes-and-emerging-products-.html

34 Bullen C, McRobbie H, Thornley S, Lin M, Laugesen M: Effect of an electronic nicotine delivery device (e-cigarette) on desire to smoke and withdrawal, user preferences and nicotine delivery: randomised cross-over trial. Tob Control 2010;19:98-103.

35 Polosa R, Caponnetto P, Morjaria JB, Papale G, Campagna D, Russo C: Effect of an electronic nicotine delivery device (e-cigarette) on smoking reduction and cessation: a prospective 6-month pilot study. BMC Public Health 2011;11:786.

36 Caponnetto P, Campagna D, Cibella F, Morjaria JB, Russo C, Polosa R: The efficacy and safety of an electronic cigarette (ECLAT) study: a prospective 12-month randomized control design study. XIV SRNT Europe Conference Helsinki, Finland, 2012. 